INPLASY

PROTOCOL

To cite: Chen et al. $1-125$ seeds with chemotherapy for progressive non-small-cell lung cancer after first-line treatment. Inplasy protocol 2021100120. doi:

10.37766/inplasy2021.10.0120

Received: 30 October 2021

Published: 30 October 2021

Corresponding author:

Yuan-Shun Xu

xuyuanshun1980@163.com

Author Affiliation:

Xuzhou Central Hospital

Support: None.

Review Stage at time of this submission: Preliminary searches.

Conflicts of interest: None declared.

\section{I-125 seeds with chemotherapy for progressive non-small-cell lung cancer after first-line treatment}

Chen, ZK 1 Xu, YS 2 .

Review question / Objective: Treatment of progressive nonsmall-cell lung cancer (NSCLC) after first-line treatment is challenging. The clinical efficacy of second-line chemotherapy for progressive NSCLC is limited. In this metaanalysis, we aim to assess the clinical effectiveness of combined I-125 seeds brachytherapy (ISB) with second-line chemotherapy for progressive NSCLC after first-line treatment.

Information sources: Relevant studies were researched in PubMed, Embase, Cochrane Library, CINK, Wanfang, and VIP (until September 2021) using the following strategy: (((((lodine-125) OR (I125)) OR (125I)) OR (brachytherapy)) AND ((lung cancer) OR (NSCLC))) AND (chemotherapy)) AND ((((recurrent) OR (failure)) OR (second line)) OR (progressive)).

INPLASY registration number: This protocol was registered with the International Platform of Registered Systematic Review and Meta-Analysis Protocols (INPLASY) on 30 October 2021 and was last updated on 30 October 2021 (registration number INPLASY2021100120).

\section{INTRODUCTION}

Review question / Objective: Treatment of progressive non-small-cell lung cancer (NSCLC) after first-line treatment is challenging. The clinical efficacy of second-line chemotherapy for progressive NSCLC is limited. In this meta-analysis, we aim to assess the clinical effectiveness of combined I-125 seeds brachytherapy (ISB) with second-line chemotherapy for progressive NSCLC after first-line treatment.

Condition being studied: Lung cancer is the leading cause of cancer mortality in the world, most (approximate $75-80 \%$ ) of which is non-small-cell lung cancer (NSCLC). For 
the patients with inoperable NSCLC, concurrent chemoradiotherapy (CCRT) is usually considered as the standard firstline treatment. When performing the CCRT, traditional external radiotherapy is commonly used. However, traditional external radiotherapy is generally associated with radiation-related complications, and potential radiological dosing is limited by the proximity of the tumor to healthy tissue and vital organs. Recently, I-125 seeds brachytherapy (ISB) has been widely used for various malignant tumor treatments. Compare to the traditional external radiotherapy, ISB has many advantages, which include ease of manipulation, direct contact with tumor surface, and sustained delivery of low-dose radiation to the tumor over an extended period of time. ISB is often used in combination with systematic chemotherapy or transcatheter arterial chemical infusion in patients with advanced NSCLC. However, most of the previous studies focused on the combined ISB and chemotherapy as the first-line treatment of advanced NSCLC. The number of studies regarding combination treatment for progressive NSCLC after first-line treatment is still limited. Therefore, a metaanalysis should be carried out to increase the statistical power of the small sample study.

\section{METHODS}

Participant or population: Patients with progressive NSCLC after first-line treatment.

Intervention: ISB with second-line chemotherapy.

Comparator: Only second-line chemotherapy.

Study designs to be included: Inclusion criteria included:(a) Type of study: comparative studies;(b) Disease: progressive NSCLC after first-line chemotherapy or CCRT;(c) Types of interventions: ISB with second-line chemotherapy versus second-line chemotherapy alone;(d) Languages:
all.Exclusion criteria included: (a) studies without control group; (b) case reports; (c) meta-analyses and reviews.

Eligibility criteria: Inclusion criteria included:(a) Type of study: comparative studies;(b) Disease: progressive NSCLC after first-line chemotherapy or CCRT;(c) Types of interventions: ISB with secondline chemotherapy versus second-line chemotherapy alone;(d) Languages: all.Exclusion criteria included: (a) studies without control group; (b) case reports; (c) meta-analyses and reviews.

Information sources: Relevant studies were researched in PubMed, Embase, Cochrane Library, CINK, Wanfang, and VIP (until September 2021) using the following strategy: ((()((lodine-125) OR (I125)) OR (125I)) OR (brachytherapy)) AND ((lung cancer) OR (NSCLC))) AND (chemotherapy)) AND ((((recurrent) OR (failure)) OR (second line)) OR (progressive)).

Main outcome(s): Tumor response, survival, and side effects.

Quality assessment / Risk of bias analysis: Potential bias was examined with the Cochrane risk of bias tool for randomized controlled trials (RCTs). The items of Cochrane risk of bias tool include performance, attrition, detection, selection, reporting, and other sources of bias. NonRCTs were assessed by the 9-point Newcastle-Ottawa scale (NOS), with studies exhibiting low, intermediate, or high levels of risk receiving scores of $\geq 7,4-6$, and $<4$, respectively. The items of NOS include selection (4 points), comparability ( 2 points), and exposure ( 3 points).

Strategy of data synthesis: RevMan v5.3 and Stata v12.0 are used for this metaanalysis. Dichotomous variables are calculated by pooled odds ratios (ORs) with 95\% confidence intervals (Cls). Pooled PFS and $O S$ are calculated by hazard ratios (HRs) with $95 \% \mathrm{Cl}$. Heterogeneity is determined by the 12 statistic and $Q$ test. 12 $>50 \%$ is defines as high heterogeneity, and then the random effect model is used; otherwise, fixed effects model is used. 
Sources of heterogeneity are evaluated by sensitivity and subgroup analyses. Egger test is used to evaluate publication bias. $P$ $<0.05$ is the threshold for publication bias significance.

Subgroup analysis: Yes.

Sensitivity analysis: Yes.

Country(ies) involved: China.

Keywords: I-125 seed; non-small-cell lung cancer; progressive; meta-analysis.

Contributions of each author:

Author 1 - Zhong-Ke Chen.

Author 2 - Yuan-Shun Xu. 